\title{
Three-Dimensional Pre-Bent Titanium Implant for Concomitant Orbital Floor and Medial Wall Fractures in an East Asian Population
}

\author{
Kyung Min Lee ${ }^{1}$, Ji Ung Park ${ }^{1,2}$, Sung Tack Kwon ${ }^{1}$, Suk Wha Kim ${ }^{1}$, Eui Cheol Jeong ${ }^{1,2}$ \\ ${ }^{1}$ Department of Plastic and Reconstructive Surgery, Seoul National University College of Medicine, Seoul; ${ }^{2}$ Department of Plastic Surgery, \\ SMG-SNU Boramae Medical Center, Seoul, Korea
}

Background The objective of this article is to evaluate clinical outcomes of combined orbital floor and medial wall fracture repair using a three-dimensional pre-bent titanium implant in an East Asian population.

Methods Clinical and radiologic data were analyzed for 11 patients with concomitant orbital floor and medial wall fractures. A combined transcaruncular and inferior fornix approach with lateral canthotomy was used for the exposure of fractures. An appropriate threedimensional preformed titanium implant was selected and inserted according to the characteristics of a given defect.

Results Follow-up time ranged from 2 to 6 months (median, 4.07 months). All patients had a successful treatment outcome without any complications. Clinically significant enophthalmos was not observed after treatment.

Conclusions Three-dimensional pre-bent titanium implants are appropriate for use in the East Asian population, with a high success rate of anatomic restoration of the orbital volume and prevention of enophthalmos in combined orbital floor and medial wall fracture cases.

Keywords Orbital fractures / Enophthalmos / Orbital implant
Correspondence: Eui Cheol Jeong Department of Plastic Surgery, SMG-SNU Boramae Medical Center, 20 Boramae 5-gil, Dongjak-gu, Seoul 156-707, Korea Tel: +82-2-870-2331 Fax: +82-2-831-2826 E-mail: ejeong.md@gmail.com

The authors wish to thank Dr. Aram Harijan for his assistance with the use of English in this manuscript.

No potential conflict of interest relevant to this article was reported.

\section{INTRODUCTION}

Orbital blowout fractures happen frequently in the context of facial trauma. The most common type of orbital wall fracture involves the floor; some of these fractures can involve concomitant fractures of the floor and the medial wall. The management of such complex fractures involving the floor and the medial wall has improved with the development of various implant materials and surgical techniques. The primary goal of all orbital wall fracture reconstructions is the restoration of the bony orbit shape and volume, the latter of which is a more significant factor for late enophthalmos deformity $[1,2]$.

Recently, three-dimensional computed tomography (CT) has become popular for the assessment of orbital fractures, and computer-assisted volume measurements are helpful in preoperative planning. These anatomic data have been used for developing many types of prefabricated alloplastic implants that complement the shape of a bony defect. Industrially pre-bent titanium implants have been designed from such anthropometric CT scan data, which closely approximate the anatomy of the human orbital floor and medial wall [3]. These pre-bent implants have become widely used in posttraumatic orbit wall re- 
constructions in Western countries. This retrospective study evaluates the accuracy of implants, reliability, and safety of the use of these implants in orbit wall reconstruction in an East Asian population in South Korea.

\section{METHODS}

The study enrolled patients who presented with medial and inferior orbital wall fractures between January 2011 and July 2013. At the conclusion of this period, 11 patients ( 9 males and $2 \mathrm{fe}$ males) had been enrolled, with a mean age of 45.5 years (range, 30-64 years). Of these, 10 patients had unilateral injuries with concomitant medial and inferior orbital wall fractures. The one remaining patient had bilateral orbital wall injuries. The mechanism of injury was a physical altercation in 5 patients, a traffic accident in 4 patients, and a fall in 2 patients. Preoperative facial bone CT scans were obtained. Functional deficits, including diplopia and gaze disturbances were evaluated by an ophthalmologist. Preoperative enophthalmos could not be evaluated in some patients because of severe periorbital swelling. Surgical indications were determined for significant diplopia based on the related fields of gaze and the fracture sizes and extent of displacement of intraorbital contents portending clinical enophthalmos. Patient demographics such as sex, age, and laterality of injury were also analyzed in the study (Table 1).

Postoperative CT images were used for comparing the herniated orbital tissue and orbit volume to the uninjured orbit. Immediate postoperative and long-term functional recovery was evaluated by comparing residual diplopia and gaze disturbances to those observed preoperatively. Postoperative complications, such as enophthalmos, late-onset diplopia, and asymmetric pu- pil locations, were also evaluated. A statistical analysis using GraphPad PRISM 5.02 (GraphPad Software, La Jolla, CA, USA) was conducted with unpaired $t$ tests. A P-value of less than 0.05 was considered statistically significant.

\section{Surgical procedure}

Under general anesthesia, the medial and inferior orbital walls were exposed through combined transcaruncular and inferior fornix (with lateral canthotomy) approaches. The periosteal incision was made with a \#15 blade, and subperiosteal dissection was carried out medially and inferiorly to reduce the soft tissue completely. After identifying the fractured segments and defects of the orbital wall, a pre-bent titanium mesh (MatrixORBITAL, Synthes, Solothurn, Switzerland) was selected according to the measured orbital rim width. A titanium implant was inserted and fixed to the inferior orbital rim with one or two titanium screws (MatrixMIDFACE, Synthes). A forced duction test was performed immediately, and passive free eye movements were confirmed prior to closure in each patient.

\section{RESULTS}

Follow-up periods ranged from 2 to 6 months (median, 4.09 months). All patients underwent surgery uneventfully, and prebent titanium mesh insertion was successful in maintaining the reduction of soft tissue herniation. None of the patients experienced postoperative ophthalmic complications.

Of the 11 patients considered, 5 patients presented with diplopia or extra-ocular muscle (EOM) limitation. One of them had traumatic optic neuropathy and presented with EOM limitation without diplopia. Postoperative diplopia was resolved within 2

Table 1. Summary of patients

\begin{tabular}{|c|c|c|c|c|c|c|c|c|c|c|c|c|}
\hline \multirow[b]{2}{*}{ No. } & \multirow[b]{2}{*}{ Gender } & \multirow[b]{2}{*}{$\begin{array}{l}\text { Age } \\
(y r)\end{array}$} & \multirow[b]{2}{*}{ Repaired fracture site } & \multirow{2}{*}{$\begin{array}{l}\text { Cause } \\
\text { of } \\
\text { injury }\end{array}$} & \multicolumn{3}{|c|}{ Preoperative evaluation } & \multirow{2}{*}{$\begin{array}{l}\text { Postoperative } \\
\text { enophthalmos } \\
\text { (on the last } \\
\text { follow-up } \\
\text { day, mm) }\end{array}$} & \multirow{2}{*}{$\begin{array}{l}\text { Period of } \\
\text { follow- } \\
\text { up (mo) }\end{array}$} & \multirow[b]{2}{*}{$\begin{array}{l}\text { Associated } \\
\text { fracture }\end{array}$} & \multirow{2}{*}{$\begin{array}{l}\text { Type of } \\
\text { implant }\end{array}$} & \multirow{2}{*}{$\begin{array}{l}\text { Trimming } \\
\text { of } \\
\text { posterior } \\
\text { end }\end{array}$} \\
\hline & & & & & Diplopia & $\begin{array}{c}\text { EOM } \\
\text { limitation }\end{array}$ & $\begin{array}{c}\text { Initial } \\
\text { enophthalmos } \\
(\mathrm{mm})\end{array}$ & & & & & \\
\hline 1 & Male & 62 & Right medial wall and floor & TA & - & - & Cannot be checked & 0 & 6 & Frontal fracture & Large & + \\
\hline 2 & Male & 36 & Right medial wall and floor & $\mathrm{TA}$ & + & + & Cannot be checked & 0.5 & 4 & Panfacial fracture & Small & + \\
\hline $3^{\text {a) }}$ & Male & 50 & Left medial wall and floor & Fall & - & + & Cannot be checked & 1 & 2 & Left NEO fracture & Small & + \\
\hline 4 & Male & 51 & Left medial wall andfloor & Assault & - & - & 0 & 0.5 & 4 & Nasal fracture & Small & + \\
\hline 5 & Male & 33 & Left medial wall and floor & Assault & - & - & 0 & 0 & 4 & - & Small & + \\
\hline 6 & Male & 30 & Left medial wall and floor & Assault & + & - & 2.5 & 0 & 2 & - & Small & + \\
\hline 7 & Male & 46 & Left medial wall and floor & Assault & + & - & Cannot be checked & 0.5 & 6 & - & Small & + \\
\hline 8 & Female & 64 & Right medial wall and floor & TA & - & - & 1 & 1.5 & 6 & Right ZMC fracture & Small & + \\
\hline 9 & Male & 35 & Left medial wall and floor & Assault & + & + & Cannot be checked & 0 & 6 & Right NEO fracture & Small & + \\
\hline 10 & Male & 44 & Left medial wall and floor & TA & + & + & 1 & 0 & 3 & Right medial wall & Small & + \\
\hline 11 & Female & 23 & Right medial wall and floor & Fall & - & - & 2.5 & 1 & 3 & Right ZMC fracture & Large & - \\
\hline
\end{tabular}

EOM, extra-ocular muscle; TA, traffic accident; NEO, naso-ethmoid-orbital; ZMC, Zygomaticomaxillary complex.

a)Patient \#3 presented with EOM limitation from blindness secondary to traumatic optic neuropathy. 


\section{Fig. 1. The change of enopthalmos}

Significant change between preoperative and postoperative Hertel exophthalmometer measurements $(\mathrm{P}<0.05)$. PreOP, preoperative value; PostOP, postoperative value.

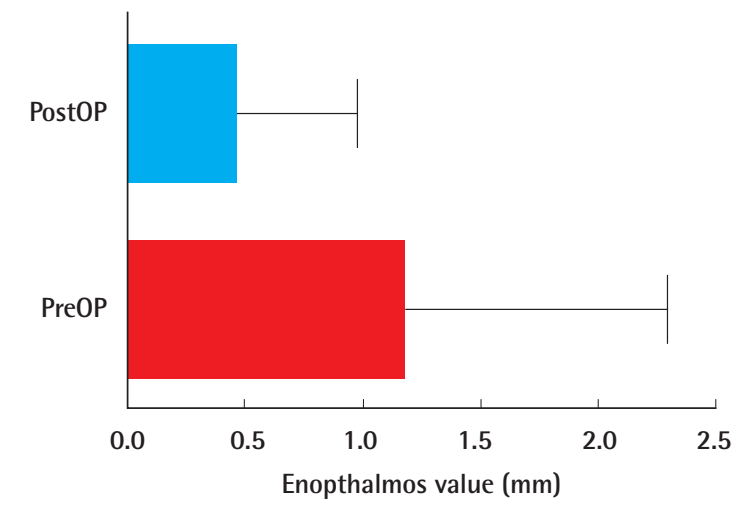

months postoperatively, and no recurrences were observed in the subsequent follow-up exams. Similarly, no EOM limitations were observed. Postoperative CT scans demonstrated appropriate positioning of intraorbital soft tissues, when compared to the contralateral orbit, and there was no implant displacement or extrusion during the follow-up period.

The mean enophthalmos showed significant improvement from preoperative to postoperative evaluation $(1.167 \pm 0.4596$ $\mathrm{mm}$ vs. $0.4545 \pm 0.1575 \mathrm{~mm} ; \mathrm{P}=0.0456$ ) (Fig. 1). No postoperative complications, such as a foreign body reaction, diplopia, EOM limitation, or visual acuity decrease, occurred during the follow-up period (Fig. 2).

\section{DISCUSSION}

In patients with orbital fractures, strong indications for operative intervention include enophthalmos greater than $2 \mathrm{~mm}$ during the first week, severe hypoglobus, or diplopia in the primary field of gaze that fails to resolve after 2 weeks, with the most common indication for surgery being the presence of a defect larger than $1 \mathrm{~cm}^{2}$. Although the timing of surgery has been a controversial issue, recently, early intervention has become preferable because late repairs were found to be associated with the adhesion and fibrosis of injured orbital tissues [4].

A modern radiological evaluation is essential for the management of orbital wall fractures. The CT scan is the best method of diagnosis, and several studies have demonstrated CT image data to be predictive of clinical enophthalmos. In South Korea, the predicted orbital volume with clinically significant enophthalmos (greater than $2 \mathrm{~mm}$ ) was found to be $2.30 \mathrm{~mL}$. This volume is sufficiently small and can be underestimated during the period of acute injury because of edema and emphysema
[5]. Therefore, enophthalmos is the most significant problem after the reduction of the concomitant orbital floor and medial wall fractures, which are almost always large defects.

Various surgical methods and implant materials have been used for the reconstruction of concomitant orbital floor and medial wall fractures. These fractures can present surgical challenges from the absent peripheral bony support for the implant and can possibly result in limited anatomical restoration of the orbital volume. The literature is replete with numerous techniques for orbital reconstruction, but the ideal choice of materials and techniques remains controversial [6].

Recent advancements in biocompatible alloplastic implants offer alternatives to traditional autologous implants. Alloplastic materials such as porous polyethylene channel implants are used widely, with low rates of infection, extrusion, and migration. More specifically, porous polyethylene channel implants offer the advantage of fibrovascular ingrowth [6]. They are relatively easy to manipulate in the surgical field but are difficult to customize according to the size and shape of a given defect. This process of trimming and bending polyethylene implants is highly operator dependent, which is a problem that affects other types of semi-rigid implants and even absorbable mesh plates.

Precise orbital wall reconstruction requires a complete understanding of the three-dimensional anatomy of the orbital wall contour. The orbital floor is known to have an initial shallow convex section behind the rim; it then inclines upward in posterior and medial directions, creating a distinct bulge behind the globe. The floor extends into the transition zone, which is an inner buttress at the junction to the lower end of the medial orbital wall (Fig. 3A). For the reconstruction of the concomitant medial wall and floor fractures, implants should be prepared to fit this exact contour, but this goal is difficult to achieve with the freehand bending of an implant.

Titanium mesh is another widely used implant in orbital trauma. Previously, these implants have also been molded freehandedly. In an effort to improve the anatomical fit, patient-specific STL models were used for intraoperative molding. However, this process is very time-consuming and costly.

Computeraided design/computeraided manufacturing generated preformed titanium orbital mesh implants have become commercially available in Western countries. They have the advantage of providing an ideal contour generated from an anthropometric analysis of more than $300 \mathrm{CT}$ scans of the human orbital skeleton. They are three-dimensionally preformed with the posterior retrobulbar bulge, which seems to play a crucial role in supporting and projecting the ocular globe and subsequently preventing postoperative enophthalmos, minimizing the need for major intraoperative manipulations of the bend and repeti- 


\section{Fig. 2. A case of combined orbital floor and medial wall fracture}

A 44-year-old man was diagnosed with right medial wall fracture and combined medial wall and floor fracture on the left side. (A) On preoperative examination, mild limitation with severe diplopia was seen during upgazing. The left eye protruded by $1 \mathrm{~mm}$ more on Hertel's exophthalmometry. (B) In the postoperative 2-month follow-up, no limitation of exra-ocular muscle movement, diplopia, or enophthalmos were noted. (C) Preoperative coronal computed tomography (CT) scan and (D) postoperative coronal CT scan.
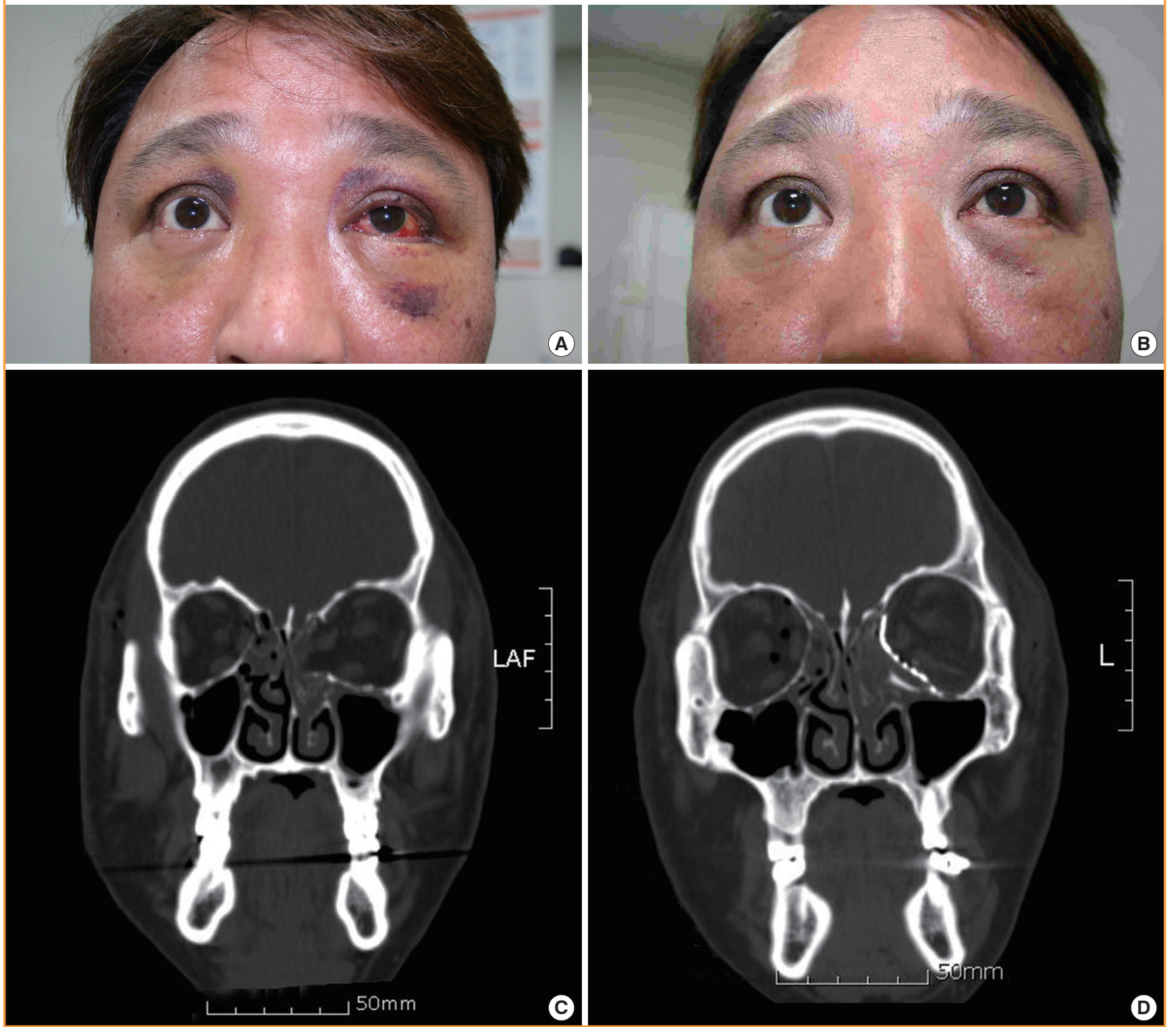

tive fittings for modification in order to reduce the operation time. These implants have already been shown to provide high contour accuracy, moderate cost, and ease of use, in comparison to patient-specific STL implants [7]. The titanium implants are radiopaque and allow for the evaluation of the intraorbital placement, which can be evaluated with an intraoperative surgical navigation system or by a postoperative CT scan.

However, these pre-bent titanium implants have been designed using anthropometric data obtained from Western/Caucasian populations, and we had reservations about whether these implants would be applicable to East Asian patients, such as the patients in South Korea, because of the anatomical differences in facial skeletons documented between Asian and Caucasian populations. Several anthropometric studies of the orbital walls in Asian populations have identified significant differences from those of Caucasians, particularly those of the relatively small orbital floor length from the orbit rim to the optic foramen [8-10]. The titanium mesh implants used in this study are produced in large and small sizes, can be chosen on the basis of the lateral orbital width and sex of the patient, and are usually used without any contouring. However, from a review of anatomical studies, we chose to use the smaller of the implants, and 


\section{Fig. 3. Three-dimensional pre-bent titanium implant for orbital fractures}

(A) Placement of Synthes MatrixMIDFACE preformed orbital plates according to the orbital landmarks. 1, orbital rim; 2, inferior orbital fissure; 3 , posterior orbital ledge; 4, transition zone between the orbital floor and the medial wall; 5, optic canal; 6, lacrimal fossa; L, left. (MatrixORBITAL Technique guide [provided $\odot$ Synthes]). (B) Two type of implants. The two images on the top show the small implants and the two below show the large ones, which are selected according to the patient's orbital anatomy and the fracture extent. The trimming of the posterior end was required in most of our cases, as marked by the small arrows at the intervening meshes.

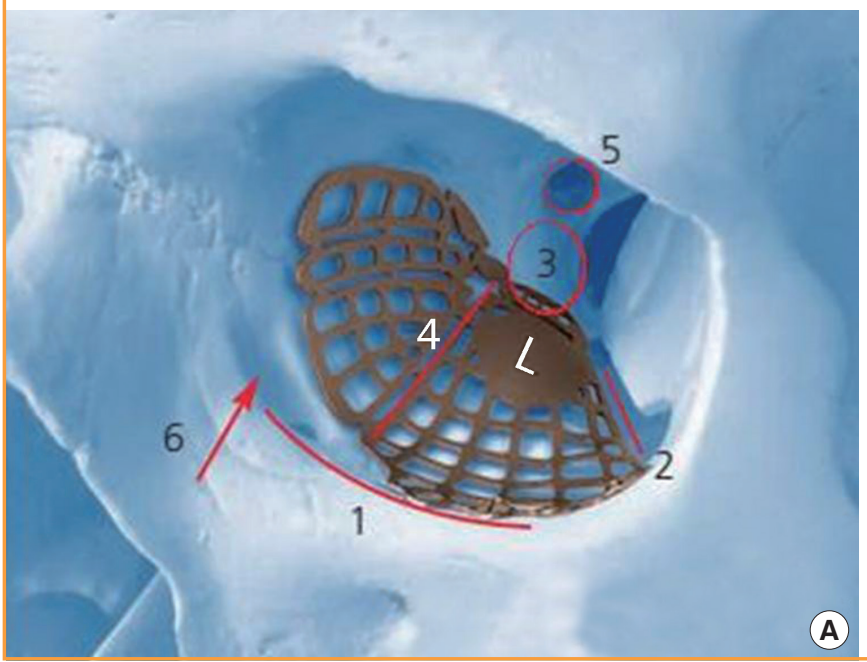

trimming the posterior end was required in most cases (Fig. 3B). When the peripheral tab needed to be trimmed, the fine intervening meshes were easily cut and left no rough edges. The implant could be inserted easily due to these smooth edges. Depending on the size of the orbital wall, we also used larger implants or implants without any trimming (Table 1).

Postoperative enophthalmos was not observed in any of the 11 cases. This demonstrated that the use of a pre-bent titanium orbital mesh implant also has the same potential for accurate post-traumatic orbital wall reconstruction with a high rate of success in East Asians. Additionally, the implant would be useful in bilateral orbital fractures with extensive anatomical unreliability. However, its application is limited in pediatric populations due to significant anatomical differences according to age [10].

In the literature, the orbital adherence syndrome has been reported after the use of titanium orbital mesh implants. Because of fibrous adhesion around the titanium mesh, a proportion of patients experienced persistent diplopia or eyelid retraction beyond two months after the orbital floor repair. This is a very rare complication that requires replacing the titanium implant with an implant made from another material or graft. However, the cause of this syndrome is still controversial. Lee and Nunery [11] hypothesized that the titanium material itself was the cause of the adhesions, but Kersey et al. [12] explained that the fracture itself frequently leads to a splitting or rupture through the periorbita, leading to adhesion by inadequate separation between the orbital contents and the bone or the implant. Thus, avoidance of titanium implants may be unwarranted if solely to

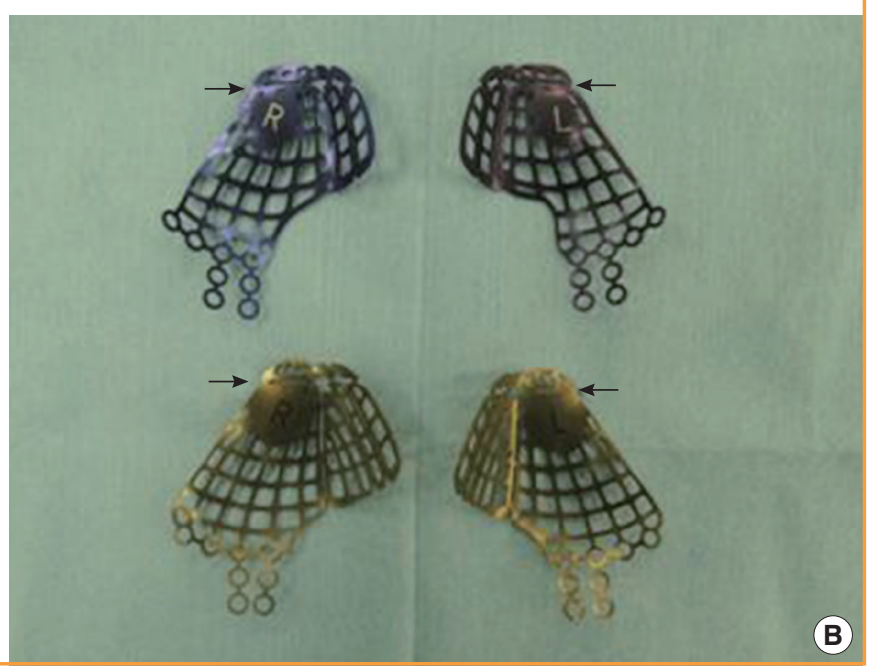

avoid a complication that may occur with or without the titanium mesh.

In this study group, the fractures were exposed with a combined transcaruncular and inferior fornix approach with lateral canthotomy. Despite the anatomical shape of these implants, insertion was relatively easy through the wide access provided by this approach. Additionally, the implant had smooth edges that did not catch soft tissues during the insertion. We believe that other approaches for exposure could be appropriate depending on the fracture and patient characteristics.

In our case series, pre-bent three-dimensional titanium implants seemed to offer safe and effective near-normal restoration of the orbital wall contour in South Korean population. Functional and aesthetic outcomes were also satisfactory, and we have concluded that these implants represent an acceptable option in the reconstruction of concomitant inferior and medial wall fractures in East Asians.

\section{REFERENCES}

1. Chen CT, Chen YR. Update on orbital reconstruction. Curr Opin Otolaryngol Head Neck Surg 2010;18:311-6.

2. He Y, Zhang Y, An JG. Correlation of types of orbital fracture and occurrence of enophthalmos. J Craniofac Surg 2012; 23:1050-3.

3. Scolozzi P, Momjian A, Heuberger J, et al. Accuracy and predictability in use of $\mathrm{AO}$ three-dimensionally preformed titanium mesh plates for posttraumatic orbital reconstruction: a 
pilot study.J Craniofac Surg 2009;20:1108-13.

4. Cole P, Boyd V, Banerii S, et al. Comprehensive management of orbital fractures. Plast Reconstr Surg 2007; 120:57S-63S.

5. Ahn HB, Ryu WY, Yoo KW, et al. Prediction of enophthalmos by computer-based volume measurement of orbital fractures in a Korean population. Ophthal Plast Reconstr Surg 2008;24:36-9.

6. Bratton EM, Durairaj VD. Orbital implants for fracture repair. Curr Opin Ophthalmol 2011;22:400-6.

7. Strong EB, Fuller SC, Wiley DF, et al. Preformed vs intraoperative bending of titanium mesh for orbital reconstruction. Otolaryngol Head Neck Surg 2013;149:60-6.

8. Metzger MC, Schon R, Tetzlaf R, et al. Topographical CTdata analysis of the human orbital floor. Int J Oral Maxillofac
Surg 2007;36:45-53.

9. Cheng AC, Lucas PW, Yuen HK, et al. Surgical anatomy of the Chinese orbit. Ophthal Plast Reconstr Surg 2008;24: 136-41.

10. Nagasao T, Hikosaka M, Morotomi T, et al. Analysis of the orbital floor morphology. J Craniomaxillofac Surg 2007;35: 112-9.

11. Lee HB, Nunery WR. Orbital adherence syndrome secondary to titanium implant material. Ophthal Plast Reconstr Surg 2009;25:33-6.

12. Kersey TL, Ng SG, Rosser P, et al. Orbital adherence with titanium mesh floor implants: a review of 10 cases. Orbit 2013;32:8-11. 